\title{
Как преодолеть разрыв между моделированием и тестированием
}

\author{
Рассказывает вице-президент и генеральный менеджер \\ направления ПО для проектирования и тестирования \\ компании Keysight Т. Катлер
}

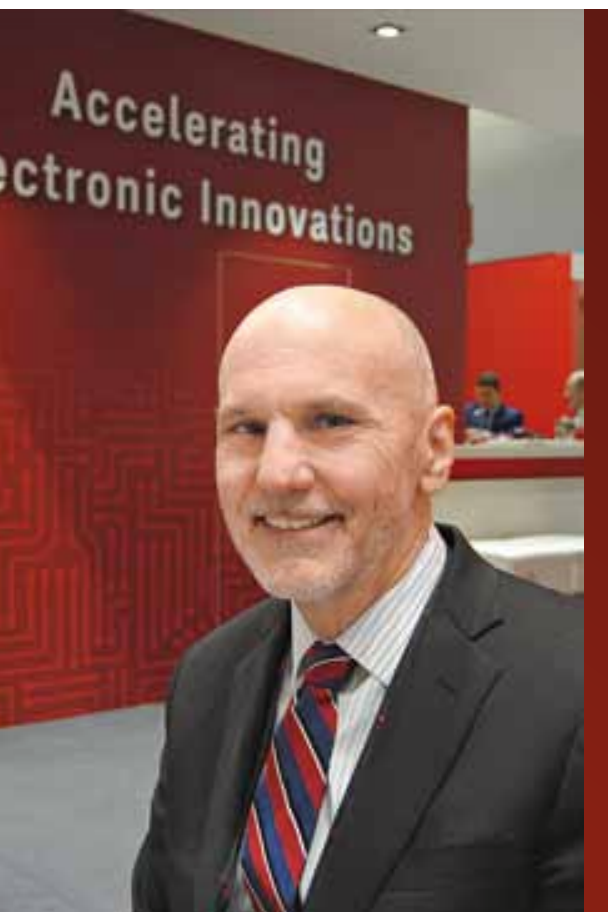

По мере повышения сложности процессов проектирования, моделирования и тестирования увеличиваются сроки и стоимость разработки. При сопоставлении данных моделирования и результатов измерения прототипов изделий возникают трудности, связанные с форматами данных на различных этапах разработки. Компания Keysight Technologies предлагает новый подход, который объединяет процессы моделирования и тестирования, решая общие задачи проектирования изделия с помощью программных технологий, позволяющих автоматизировать процессы анализа данных и ускорить оценку результатов моделирования. Об инструментах и технологиях, повышающих эффективность разработки изделий, и путях развития российского рынка нам рассказал вице-президент и генеральный менеджер направления ПО для проектирования и тестирования компании Keysight Тодд Катлер (Todd Cutler).

Господин Катлер, каковы последние тенденции в сфере развития программных технологий компании Keysight?

Прежде всего следует выделить три основных направления, которые компания Keysight рассматривает как наиболее перспективные сегодня. Первое 5G-системы - сфера, в которой мы начали работать одними из первых на рынке и добились существенных успехов с точки зрения разработки средств прототипирования и тестирования. Второе направлениеавтомобильные системы, куда мы также инвестируем значительные ресурсы. И третье - программное обеспечение, охватывающее все этапы создания продукта: от проектирования до измерения характеристик. Keysight уделяет особое внимание этому направлению как одному из важнейших факторов повышения конкурентоспособности и усиления позиций компании на рынке.

Обычно проектирование нового устройства начинают с компьютерного моделирования, чтобы убедиться в правильности выбора проектного решения.
После создания прототипа устройства нужно измерить его характеристики и сравнить их с данными, полученными при моделировании. Однако здесь возникают трудности, поскольку инструменты моделирования и тестовое ПО «говорят» на разных языках-данные в этих системах представлены в различных форматах. Разработчики вынуждены тратить немало времени и прилагать много усилий, чтобы сопоставить модель и реальное поведение системы. Компания Keysight поставила перед собой задачу преодолеть разрыв между данными моделирования и результатами измерений, по сути, соединить виртуальный и реальный мир, чтобы помочь своим клиентам сэкономить время и деньги. С этой целью мы разработали новую программную платформу, которая получила название PathWave.

Расскажите, пожалуйста, подробнее о платформe PathWave.

PathWave - это открытая масштабируемая программная платформа разработки, которая объединяет процессы проектирования, тестирования 
и верификации. Она включает в себя инструменты проектирования, пО управления измерительными приборами и прикладное ПО, интегрированные в единой программной среде. Платформа обеспечивает общие форматы данных и интерфейсы управления, предоставляет инженерам широкие возможности сбора, обмена и анализа данных испытаний и измерений.

Ключевой принцип построения новой платформы общие форматы данных на разных этапах разработки. Чтобы можно было использовать данные проектирования в измерительной среде (и наоборот), мы стандартизировали не только процедуру хранения данных моделирования и тестирования, но и формат, в котором они изначально генерируются.

Платформа поддерживает любое количество специальных тестов и приложений, используя программные и аппаратные средства, включенные в маршрут проектирования. При создании ПО для проектирования, тестирования и анализа данных Keysight сотрудничает с ведущими вендорами в сфере САПР, включая Cadence, Mentor и Synopsys, а также компаниями, paботающими в области измерений. Открытый АРІ-интерфейс платформы позволяет не только клиентам, но и любым сторонним организациям создавать на его основе собственное ПО. Кроме того, благодаря открытому исходному коду наши клиенты могут модифицировать те модули платформы, которые им нужны для работы.

Еще одна важная особенность платформы PathWave - масштабируемость. Ее можно будет запускать на ПК, в том числе на Linux-машине, на сервере, а также на планшете или даже смартфоне. Предусмотрена возможность создания облачной версии этой платформы.

В России идет дискуссия о том, может ли моделирование заменить физическое тестирование. Каково ваше мнение?

я абсолютно уверен, что не может. Точность моделирования зависит от того, как много параметров включено в модель. Но если вы задействуете огромное число характеристик, то процесс моделирования займет слишком много времени. Крометого, реальная система слишком сложна, чтобы можно было учесть все факторы, влияющие на ее поведение. Поэтому на этапе моделирования разработчики всегда допускают определенное упрощение. Инженеру нужно хорошо разбираться в современных симуляторах и инструментах разработки, чтобы понимать все тонкости ПО и постараться максимально использовать его возможности

Следует учитывать, что моделирование не настолько эффективно, чтобы с первой попытки обеспечить желаемый результат. Конечно, хотелось бы, но реальные системы настолько сложные, что этого добиться практически невозможно. Некоторым разработчикам удается получить удовлетворительный результат, в частности со второй попытки. Однако есть примеры того, как инженерам приходилось до десяти раз вносить изменения в прототип по результатам моделирования, прежде чем он стал работать корректно. Сегодня большинство специалистов ограничиваются двумя-тремя-четырьмя итерациями. Я думаю, что это показывает важность корректного выполнения моделирования: вы можете сократить число итераций, необходимых для создания работающего прототипа.

\section{Keysight уделяет особое внимание развитию направления ПО как одному из важнейших факторов повышения конкурентоспособности и усиления позищий компании на рынке}

Я предполагаю, что ценность моделирования различается в зависимости от направлений разработки, например, Свч ис или печатные платы. Для каких областей моделирование максимально эффективно?

Да, вы правы, ценность моделирования различается. Чтобы создать прототип ИС, нужны несколько месяцев и значительные финансовые затраты. А для разработки, например, печатной платы требуется пара дней. Поэтому и значимость моделирования для разработчиков ИС значительно выше, чем для разработчиков печатных плат, ведь стоимость и время создания прототипа печатной платы намного меньше. Кроме того, цена ошибки для разработчиков ИС значительно выше, чем для разработчиков печатных плат. Если рассматривать область САПР в целом, то стоимость бизнеса в полупроводниковой отрасли в несколько раз превосходит стоимость бизнеса в области печатных плат именно потому, что цена некорректного решения на стадии создания Ис очень высока.

y Keysight есть мощные средства для проектирования СВЧ ИС. Какие еще виды инструментов для создания ИС предлагает ваша компания?

Это зависит от того, какие схемы предстоит разрабатывать. Наша сильная сторона - инструменты моделирования аналоговых схем и схем смешанного сигнала. Что касается цифровых схем, то в этой сфере наше предложение ограничено. У нас есть ряд 
инструментов для разработки высокоскоростных цифровых интерфейсов, применяемых для коммуникаций между кристаллами или печатными платами, но для создания цифровых ИС нужно пользоваться инструментами других компаний.

\section{Компания Keysight nоставила перед} собой задачу, по сути, соединить виртуальный и реальный мир, чтобы помочь своим кииентам сэкономить время и деньги. С этой целью мы разработали новую программную
платформу PathWave

В микроэлектронике технологии сильно влияют на характеристики чипа или системы в корпусе. Есть ли на рынке инструменты, учитывающие особенности технологии конкретной фабрики, например TSMC или другой компании, или все инструменты - универсальные и подходят для любого технологического процесса?

Любой хороший инструмент помогает в большинстве случаев, когда используется точная модель транзистора или другого устройства. Долгое время мы были лидером в области измерения характеристик транзисторов, резисторов, конденсаторов, создавали модели этих компонентов на уровне кристалла. Как правило, все технологические особенности учитываются в моделях, которые разрабатываются компаниями-производителями. Каждый производитель рекомендует для применения те или иные модели, оптимально подходящие для данного техпроцесса. кроме того, модель может быть создана разработчиком для какого-либо специфического технологического процесса. Следует помнить, что для получения хороших результатов моделирования нужно использовать достаточно точную модель.

Сегодня при моделировании схем необходимо учитывать не только электрические характеристики, но и другие факторы, в частности механические параметры, радиационные эффекты и т. п. Как решается проблема сочетания в одном маршруте проектирования различных видов САПР (механических, температурных и т.д.)?

По-видимому, вы имеете в виду программы так называемого мультифизического моделирования, которые учитывают механические нагрузки, тепловые режимы и другие факторы. Команды разработчиков трудятся над решением этих проблем. Мы также занимаемся подобными вещами. Например, предлагаем своим клиентам инструмент для моделирования электромагнитных полей, который называется EMPro, а также инструмент для электротермического анализа HeatWave. Мы не работаем в области механических САПР или инструментов для анализа радиационных эффектов - это вне нашей компетенции. я бы не сказал, что это широко востребованные на рынке продукты, но ими занимаются некоторые компании-разработчики. Это достаточно нишевой сектор, например, САПР для радиационного анализа применяют в аэрокосмической отрасли. Следует отметить, что модели для радиационного или температурного анализа отличаются высокой сложностью и для быстрого получения результата моделирования требуются значительные вычислительные ресурсы.

Как вы оцениваете российский рынок и перспективы бизнеса компании в нашей стране?

Наш бизнес в России подвержен цикличности. Еще несколько лет назад наши дела шли хорошо, мы много инвестировали в развитие деятельности компании. Сейчас ситуация ухудшилась. Честно говоря, у меня нет четкого понимания причин этого. Наше присутствие на российском рынке достаточно широко, и мы продолжаем в него инвестировать. На мой взгляд, в России нужно развивать бизнес-среду. У нас в компании работает огромное количество талантливых российских инженеров, в вашей стране замечательная система образования. Но вопрос заключается в том, что нужно построить бизнес в России так, чтобы он носил международный характер. Ваши продукты не знают за пределами страны, я не могу пойти в магазин и купить продукт, спроектированный или произведенный в России. Но основа для построения успешного бизнеса в стране есть. Кстати, мы помогаем его развитию с помощью такого рода публикаций в вашем журнале.

Еще одним способом развития рынка могло бы стать сотрудничество с российскими компаниями в области проектирования, а также создание в стране исследовательских лабораторий, работающих как часть американских или других компаний. Собственно, сейчас нередко так и происходит. я знаю несколько компаний, для которых разработки ведутся в России. Также не стоит забывать про инвестиции в вашу электронную отрасль, однако эта задача напрямую зависит от решения политических вопросов.

Спасибо за интересный рассказ.

СТ. Катлером беседовал Ю. Ковалевский 